\title{
Passive imaging and detection in cluttered media
}

\author{
Josselin Garnier \\ Laboratoire de Probabilités et Modèles Aléatoires \\ \& Laboratoire Jacques-Louis Lions, \\ Université Paris 7, 2 Place Jussieu, 75251 Paris Cedex 5, France \\ Email: garnier@math.jussieu.fr \\ Knut Solna \\ Department of Mathematics, \\ University of California at Irvine, Irvine, CA 92697, USA \\ Email: ksolna@math.uci.edu
}

\begin{abstract}
In this paper we consider passive sensor imaging with ambient noise sources by suitably migrating the cross correlations of the recorded signals. We use an imaging functional that relates to the classic Kirchhoff migration functional. We analyze the properties of the imaging functional in the highfrequency regime which shows that it produces sharp images. We identify the scaling assumptions that allow us to image respectively, the support of the random sources and the medium variations when these may come in the form of clutter. Numerical simulations confirm the theoretical predictions.
\end{abstract}

\section{INTRODUCTION}

In a number of physical situations there are background or ambient noise present in a scenery of interest. This is for instance the case in the earth's crust where microseisms and also signals due to ocean swells can propagate over long distances. Thus, even without using an active source a measurement array would detect signals. The surprising fact is that these signals, generated by ambient noise, actually can be used for imaging. By cross correlating such noisy measurement one can in particular estimate the Green's function of the wave equation in an inhomogeneous medium. The situation we shall consider is the one in which noise sources with unknown spatial support emit stationary random signals, that propagate into the medium and are recorded at a set of observation points. The cross correlation of the recorded signals has been shown to provide a reliable estimate of the Green's function and the travel time between the observation points [10], [11]. Mathematical analyses of cross correlation of noisy signals have been carried out for instance in [1], [5], [7], [8], showing the importance of medium heterogeneity and the delicate interaction of phenomena on different scales. A central aspect is to identify how the statistics of the signal on the microscale relate to the macroscale. The travel time estimates can be used for background velocity estimation [6]. Tomographic travel time velocity analysis, based on cross correlations, was applied successfully for surface-wave velocity estimation in Southern California [12], in Tibet [14], and in the Alps [13]. In [4] inter-period differentials in cross-correlations were used to identify seismic velocity variations which were correlated to subsequent eruptions. Cross correlations of noisy signals recorded by a passive sensor array can also be processed in order to image the medium. The idea presented in [8] is to backpropagate the cross correlation matrix by applying a simple Kirchhoff migration (KM) functional, which is a technique widely used in active seismic imaging [2]. We continue this analysis here and identify explicitly the form of the Kirchhoff functional in the case with imaging of incoherent medium clutter. We shall assume a here a twodimensional medium and a passive sensor array on the surface of a halfspace where clutter and ambient noise sources are located. Our goal is to image the source locations and the scatterers embedded in the medium, the variations of the speed of propagation around a known constant background velocity, using the cross correlation matrix of the recorded signals. We consider an imaging functional related to the classic Kirchhoff imaging scheme in that it is a function of the cross correlation matrix. The function we consider here is obtained by summing the cross correlations evaluated at some special differential travel times over a limited offset range. By a high-frequency asymptotic analysis and by numerical simulations we will show that this imaging functional produces sharp images. In Section II the schematic wave propagation scenario that we shall consider is described. Then, we consider in Section III the problem of the localization of the noise sources and move on in Section IV to the problem of the localization of scatterers embedded in the medium.

\section{Waves, Ambient Noise And Clutter}

We consider the solution $u$ of the non-dimensionalized scalar wave equation in a two-dimensional inhomogeneous medium with background velocity $c(\boldsymbol{x})$ :

$$
\frac{1}{c^{2}(\boldsymbol{x})} \frac{\partial^{2} u}{\partial t^{2}}-\Delta_{\boldsymbol{x}} u=n(t, \boldsymbol{x})
$$

with $\boldsymbol{x}=(x, z)$. The source term $n(t, \boldsymbol{x})$ models a random ambient noise sources and is supported in the halfspace $z<0$. It is assumed to be a stationary process in time and being deltacorrelated in space, so that its autocorrelation function has the form

$$
\left\langle n\left(t_{1}, \boldsymbol{y}_{1}\right) n\left(t_{2}, \boldsymbol{y}_{2}\right)\right\rangle=F\left(t_{2}-t_{1}\right) K\left(\boldsymbol{y}_{1}\right) \delta\left(\boldsymbol{y}_{1}-\boldsymbol{y}_{2}\right) .
$$

Here the brackets stand for the statistical averaging with respect to the distribution of the sources. The function $F$ has support $\lambda_{0} / c_{0}$ with $\lambda_{0}$ playing the role of source wavelength in this problem, moreover, we assume that its Fourier transform, $\hat{F}$, is smooth and vanish at the origin, moreover, is of rapid decay at infinity. The compactly supported function $K(\boldsymbol{y})$ is the source region and we seek first to estimate this. 
The clutter or medium perturbation comprise two parts. The first is smooth with slow variation and is modeled by a smooth and deterministic function. The second is rapid and with random fluctuations and is modeled by a stochastic field. We shall assume that the background velocity is of the form

$$
\frac{1}{c^{2}(\boldsymbol{x})}=\frac{1}{c_{0}^{2}}\left\{1+h(\boldsymbol{x})\left(\sigma_{0}+\sigma_{1} \nu(\boldsymbol{x})\right)\right\},
$$

where $\sigma_{0}$ and $\sigma_{1}$ are the magnitudes of the smooth and random parts of the medium clutter. The stationary random process $\nu$ has mean zero, variance one, and correlation length $\ell_{0}$ defined by

$$
\ell_{0}^{2}=\int \mathbb{E}[\nu(\mathbf{0}) \nu(\boldsymbol{x})] \mathrm{d} \boldsymbol{x},
$$

where $\mathbb{E}$ stands for the averaging with respect to the distribution of the random medium. The smooth and deterministic function $h$ gives the support of the clutter which is assumed to be compactly supported, connected and located in the region $z<0$, moreover, disjoint from the random source support, we also seek to estimate this clutter support. Note that in this paper we focus on the rough clutter component so that $\sigma_{0}=0$.

Regarding problem scaling we shall assume

$$
\lambda_{0} \ll L, \quad \sigma_{0} \ll\left(\frac{\lambda_{0}}{\mathcal{L}}\right)^{3 / 2}, \quad \sigma_{1} \ll\left(\frac{\lambda_{0}}{\ell_{0}^{2 / 3} \mathcal{L}^{1 / 3}}\right)^{3 / 2},
$$

where $\mathcal{L}$ is the characteristic dimension of the scattering region and $L$ is the typical propagation distance.

Next, we describe the pre-processing of the data to "create signal from noise" via forming correlations. The (one dimensional) receiver array is at the surface $z=0$ with inter-element spacing smaller than the wavelength so that we can assume continuous measurements. We form the empirical cross correlation of the signals recorded at two receivers at $\boldsymbol{x}_{1}$ and $\boldsymbol{x}_{2}$ by:

$$
C_{T}\left(\tau, \boldsymbol{x}_{1}, \boldsymbol{x}_{2}\right)=\frac{1}{T} \int_{0}^{T} u\left(t, \boldsymbol{x}_{1}\right) u\left(t+\tau, \boldsymbol{x}_{2}\right) \mathrm{d} t .
$$

As shown in [8], it is a statistically stable quantity in the sense that it converges to its statistical mean (with respect to the random sources), $C\left(\tau, \boldsymbol{x}_{1}, \boldsymbol{x}_{2}\right)$, when the integration time $T$ goes to infinity. Writing the recorded field in terms of the Green's function and the source term, and using the particular form of the autocorrelation function of the sources, we obtain the expression:

$$
\begin{aligned}
C\left(\tau, \boldsymbol{x}_{1}, \boldsymbol{x}_{2}\right)= & \frac{1}{2 \pi} \iint \mathrm{d} \boldsymbol{y} \mathrm{d} \omega \hat{F}(\omega) K(\boldsymbol{y}) \\
& \times \overline{\hat{G}}\left(\omega, \boldsymbol{x}_{1}, \boldsymbol{y}\right) \hat{G}\left(\omega, \boldsymbol{x}_{2}, \boldsymbol{y}\right) e^{-i \omega \tau},
\end{aligned}
$$

where $\hat{G}$ is the outgoing time-harmonic Green's function. When the background velocity is homogeneous $c(\boldsymbol{x})=c_{0}$, the Green's function is given in terms of the zeroth order Hankel function of the first kind. Using the asymptotic form of the Hankel function we find that for $|\omega||\boldsymbol{x}-\boldsymbol{y}| / c_{0} \gg 1$ :

$$
\hat{G}(\omega, \boldsymbol{x}, \boldsymbol{y})=\frac{\sqrt{c_{0}}}{2 \sqrt{2 \pi}} \frac{e^{i \operatorname{sgn}(\omega) \pi / 4}}{\sqrt{|\omega||\boldsymbol{y}-\boldsymbol{x}|}} \exp \left(i \frac{\omega}{c_{0}}|\boldsymbol{y}-\boldsymbol{x}|\right) .
$$

In our context the central part of this Green's function is the phase associated with the travel time: $\mathcal{T}(\boldsymbol{x}, \boldsymbol{y})=|\boldsymbol{y}-\boldsymbol{x}| / c_{0}$.

Formally, we find that if we consider the situation with point support for the sources at $\boldsymbol{y}^{c}$ and approximate the Green's function by its phase component then:

$$
C\left(\tau, \boldsymbol{x}_{1}, \boldsymbol{x}_{2}\right) \approx F\left(\tau-\left[\mathcal{T}\left(\boldsymbol{y}^{c}, \boldsymbol{x}_{2}\right)-\mathcal{T}\left(\boldsymbol{y}^{c}, \boldsymbol{x}_{1}\right)\right]\right) .
$$

This suggests the Kirchhoff imaging functional at a search point $\boldsymbol{y}^{S}$ and recivers at $\boldsymbol{x}_{1}, \ldots, \boldsymbol{x}_{N}$ as being defined by

$$
\mathcal{I}^{\mathrm{KM}}\left(\boldsymbol{y}^{S}\right)=\sum_{j, l=1}^{N} C_{T}\left(\mathcal{T}\left(\boldsymbol{y}^{S}, \boldsymbol{x}_{l}\right)-\mathcal{T}\left(\boldsymbol{y}^{S}, \boldsymbol{x}_{j}\right), \boldsymbol{x}_{j}, \boldsymbol{x}_{l}\right) \text {. }
$$

We assume that the receivers are uniformly distributed in the interval $[-A / 2, A / 2]$ at the surface $(z=0)$ and separated by $\delta x=A / N<\lambda_{0}$. In the frequency domain, the imaging functional (8) means that a matched filter is applied in order to compensate for the phase term in the high-frequency expression of the cross correlations and is the form which we will use in subsequent sections.

\section{LOCALIZING THE NOISE SOURCES FROM CORRELATIONS}

We consider the scaling assumptions described in Equation 4. This allow us to carry out a high-frequency analysis of the Kirchhoff imaging functional. A main technical tool that we use is the stationary phase approximation which approximates integrals by identifying the main contribution as coming from points of stationarity of the phase. This approximation is accurate in a regime of very small wavelength $\lambda_{0}$, when the phase fluctuates fast due to the high frequencies. Physically the stationary points corresponds to directions of information propagation.

We then find that, in the case with homogeneous medium, so that $\sigma_{0}=\sigma_{1}=0$, the Kirchhoff imaging functional gives an image of the source support. Approximating sums by integrals we can write :

$$
\mathcal{I}^{\mathrm{KM}}\left(\boldsymbol{y}^{S}\right)=\frac{N^{2}}{16 \pi^{2}} \iint \mathrm{d} \boldsymbol{y} \mathrm{d} \omega \hat{F}(\omega) K(\boldsymbol{y}) \mathcal{K}_{\mathrm{KM}}\left(\omega, \boldsymbol{y} ; \boldsymbol{y}^{S}\right)
$$

where the refocusing kernel is

$$
\begin{array}{r}
\mathcal{K}_{\mathrm{KM}}\left(\omega, \boldsymbol{y} ; \boldsymbol{y}^{S}\right)=\frac{c_{0}}{A^{2}|\omega|} \iint_{-A / 2}^{A / 2} \frac{\mathrm{d} x_{1} \mathrm{~d} x_{2}}{\sqrt{\left|\boldsymbol{y}-\boldsymbol{x}_{1}\right|\left|\boldsymbol{y}-\boldsymbol{x}_{2}\right|}} \\
\times e^{\left.i \omega\left[\mathcal{T}\left(\boldsymbol{y}^{S}, \boldsymbol{x}_{1}\right)-\mathcal{T}\left(\boldsymbol{y}, \boldsymbol{x}_{1}\right)\right)-\mathcal{T}\left(\boldsymbol{y}^{S}, \boldsymbol{x}_{2}\right)+\mathcal{T}\left(\boldsymbol{y}, \boldsymbol{x}_{2}\right)\right]},(10
\end{array}
$$

with $\boldsymbol{x}_{j}=\left(x_{j}, 0\right)$. We next comment on the refocusing kernel.

Assume $\boldsymbol{y}^{S}=L(\cos (\theta), \sin (\theta))$ with $L \gg 1$ and $\boldsymbol{y}=$ $\boldsymbol{y}^{S}+\left(y_{1}, y_{2}\right)$, we have

$\mathcal{K}_{\mathrm{KM}}\left(\omega, \boldsymbol{y} ; \boldsymbol{y}^{S}\right) \sim \frac{c_{0}}{|\omega| L} \operatorname{sinc}^{2}\left(\frac{\omega A\left[y_{1}-y_{2} \sin (2 \theta) / 4\right]}{2 c_{0} L}\right)$.

Thus, with the source just below the array (i.e. $\theta=0$ ) with $A \ll L$ we see that we have a good resolution in the lateral direction, but very poor resolution in the depth direction. Indeed the longitudinal resolution is given by the Rayleigh 
resolution, $\lambda_{0} L / A$. However, with an aperture of order the distance to the clutter to be imaged the Kirchhoff imaging functional will give good resolution also in the depth direction via subarray processing.

Thus, indeed this functional focuses at the support of the sources as determined by $K$. This process amounts to using differential travel time estimates, as given by the correlations, to construct the image. We remark that assuming free boundary conditions, modeling for instance the air-ground interface, may yield enhanced depth resolution due to reflections from the interface.

We next carry out numerical simulations in an open twodimensional homogeneous medium. The source distribution is modeled by a collection of point sources randomly distributed in a compactly supported domain. The point sources emit stationary random signals with Gaussian statistics and mean zero. The power spectral density is $\hat{F}(\omega)=|\omega| \exp \left(-\omega^{2} / 400\right)$. The background velocity is one. Therefore the carrier frequency is $\omega_{0} \simeq 14$ and the carrier wavelength is $\lambda_{0} \simeq 0.45$. There are 200 sensors at the surface $z=0$ separated by $\delta x=0.125$ on the interval $x \in(-12.5,12.5)$. This corresponds to a dense array for (almost) all frequencies in the bandwidth. There are 300 independent point sources randomly distributed in the three rectangles $[-1.5,-1] \times[-9,-8],[-0.5,0] \times[-10,-9]$, and $[0.5,1] \times[-11,-10]$. The reconstruction grid is a $100 \times 100$ uniform grid on the square $[-4,4] \times[-15,-5]$.

In Figure 1 we can observe the good resolution obtained with the Kirchhoff migration method and that we indeed can identify the source region from the sensor array correlations.

\section{Detecting Medium Clutter}

We now consider imaging of the scattering region. In this case the noise from the sources will be scattered by the clutter which then acts as secondary sources. Differential travel times estimates can now be associated with these secondary sources via the correlations and we can use the Kirchhoff imaging functional as before to identify the image. We remark that the functional also will focus at the support of the noise sources. An important aspect of this configuration is to what extent the scatter will redirect signal energy. If we have smooth scatterers with only very weak refraction then there should be a "ray" going from the measurement array through the point we want to image to a point in the ambient noise region, otherwise, the measurements will not give a good image of this point. The situation with smooth clutter, either a deterministic component $\left(\sigma_{0} \neq 0\right)$ or a random component with $\ell_{0} \gg \lambda_{0}$ can in fact best be analyzed via the Radon transform in the context of wide aperture as the Kirchhoff functional can be expressed in terms of products of line integrals of respectively clutter and source distributions. Thus, identification of the clutter region in this case require the prior identification of the source region. We shall not focus on this case here, see [9].

We consider thus here the situation with $\sigma_{0}=0$ and $\sigma_{1} \neq 0$ and $\ell_{0} \ll \lambda_{0}$ which corresponds to a situation with strong redirection of signal energy. Then we find that the expected

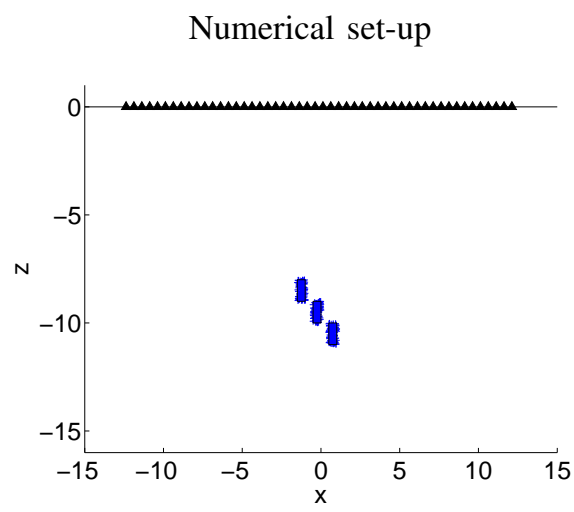

Kirchhoff Migration Image

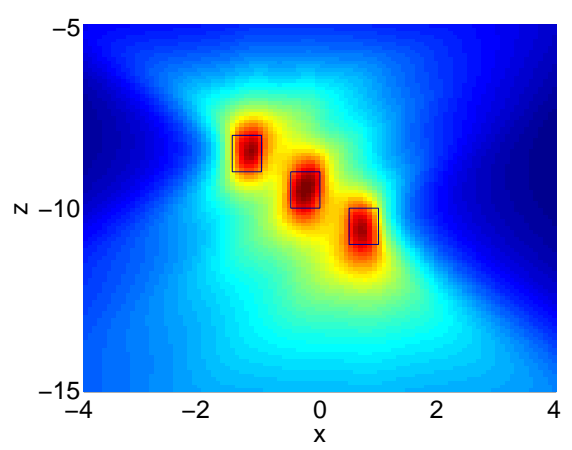

Fig. 1. Imaging the sources when the spatial source distribution is compactly supported in three rectangles. The numerical set-up is plotted in the top figure, where the triangles represent the sensors at the surface $z=0$ and the independent point sources lie in the rectangles in the subsurface. The image obtained with the Kirchhoff method is plotted in the bottom figure.

value of the Kirchhoff imaging functional, $\mathbb{E}\left[\mathcal{I}^{\mathrm{KM}}\left(\boldsymbol{y}^{S}\right)\right]$, will focus on the clutter in addition to the random sources and in fact give an image of $h$ in the case with large aperture. We can write explicitly, using a Born approximation, for $\boldsymbol{y}^{S}$ in the vicinity of the clutter:

$$
\begin{array}{r}
\mathbb{E}\left[\mathcal{I}^{\mathrm{KM}}\left(\boldsymbol{y}^{S}\right)\right]=\frac{N^{2}}{16 \pi^{2}} \iint \mathrm{d} \boldsymbol{y} \mathrm{d} \omega \hat{F}(\omega)|\omega|^{3} H(\boldsymbol{y}) \\
\times \mathcal{K}_{\mathrm{KM}}\left(\omega, \boldsymbol{y}, \boldsymbol{y}^{S}\right),
\end{array}
$$

where the function

$$
H(\boldsymbol{y})=\frac{\sigma_{1}^{2} l_{0}^{2}}{8 \pi c_{0}} h^{2}(\boldsymbol{y})\left(\int \frac{K(\boldsymbol{z})}{|\boldsymbol{z}-\boldsymbol{y}|} \mathrm{d} \boldsymbol{z}\right)
$$

has the same support as $h$. Thus, by comparison with (9) we see that the resolution scaling will be similar as when imaging the source distribution.

In the above equation $\mathbb{E}$ represents expectation with respect to the distribution of the clutter. In the high-frequency limit one can show that this imaging functional indeed is statistically stable so that the imaging functional computed for a certain realization of the clutter gives an estimate of the clutter support, that is, $\mathbb{E}\left[\mathcal{I}^{\mathrm{KM}}\right] \approx \mathcal{I}^{\mathrm{KM}}$. Verifying this involves a fourth order moment calculation of the data. Clearly, this property of statistical stability is important for imaging in a practical setting. 


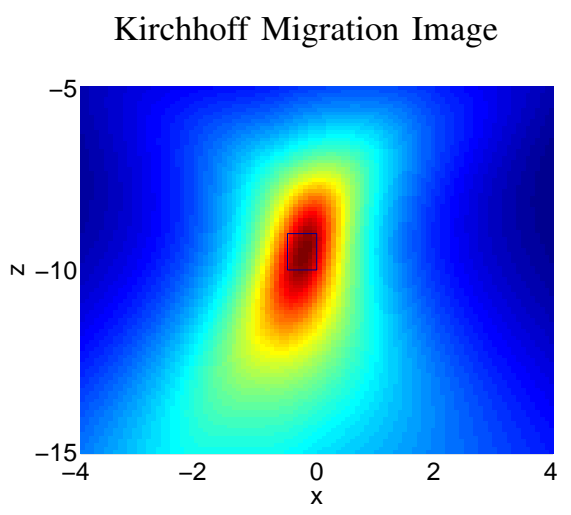

Fig. 2. The Kirchhoff medium image when there is one point source located at $(-20,-100)$. The sensors are at the surface $z=0$ and the scatterers lie in a rectangle in the subsurface.

In the numerical simulations the background velocity is one and there are 200 sensors at the surface $z=0$ separated by $\delta x=0.125$ on the interval $x \in(-12.5,12.5)$. There is one noise point source located at $(-20,-100)$. There are 100 point scatterers with reflectivities 0.1 randomly distributed with the uniform distribution in the rectangle $[-0.5,0] \times[-10,-9]$. The power spectral density of the noise sources is here $\hat{F}(\omega)=$ $|\omega|\left(1+\omega^{2}\right)^{-3 / 2} \exp \left(-\omega^{2} / 400\right)$, so that $|\omega|^{3} \hat{F}(\omega)$ is close to the spectral density used in the simulations of Section III and the noise signals scattered by the scattering region have almost the same spectrum as the one of the noise signals emitted by the sources in Section III.

The result is shown in Figure 2 shows that even with only one noise source we can image the scatterer. One can see, however, that the direction of the energy flux coming from the source point has an impact on the image (the energy flux has approximately the direction of the vector $(1,5)$ since the point source is located at $(-20,-100))$.

\section{Conclusions}

In this paper we have discussed a promising passive imaging technique: migration of cross correlation of noisy signals. We have described how classic migration techniques generalize to the case of ambient noise and correlation based imaging. The important analytic tool that we have used is highfrequency analysis which in fact yield simple characterization for complex quantities defined in terms of quadratic and forth order functionals of the data. The framework that we have considered is not limited to the high-frequency waves case, however, this is a convenient assumption to identify explicit expressions for the imaging functionals and associated resolution.
We stress also that the formulation is high-frequency in that the wavelength is short relative to the total propagation distance, however, as we discussed above the wave length can be large relative to the target, that is, the medium clutter to be imaged. Important generalizations of the techniques set forth are in particular: i) the case with three-dimensional media and non-homogeneous, but slowly varying background which can be analyzed via WKB techniques. ii) considering higher order, that is fourth order correlations, see [8]. iii) construction of optimized imaging functionals, both with respect to resolution and statistical stability, exploiting in particular fast transform in regular sampling configurations, see [9]. iv) detailed analysis of signal versus noise relations and the design of explicit detection tests. v) other source and measurement configurations, we mention that we here considered the socalled backlight configuration.

\section{REFERENCES}

[1] C. Bardos, J. Garnier, and G. Papanicolaou, Identification of Green's functions singularities by cross correlation of noisy signals, Inverse Problems, 24 (2008), 015011.

[2] N. Bleistein, J. K. Cohen, and J. W. Stockwell Jr, Mathematics of multidimensional seismic imaging, migration, and inversion, Springer Verlag, New York, 2001.

[3] L. Borcea, G. Papanicolaou, and C. Tsogka, Theory and applications of time reversal and interferometric imaging, Inverse Problems, 19 (2003), pp. S134-S164.

[4] F. Brenguier, N. M. Shapiro, M. Campillo, V. Ferrazzini, Z. Duputel, O. Coutant, and A. Nercessian, Toward forecasting volcanic eruptions using seismic noise, arXiv:0706.1935v1, (2007).

[5] Y. Colin de Verdière, Mathematical models for passive imaging. I: General background, arXiv:math-ph/0610043v1 (2006).

[6] A. Curtis, P. Gerstoft, H. Sato, R. Snieder, and K. Wapenaar, Seismic interferometry - turning noise into signal, The Leading Edge, 25 (2006), pp. 1082-1092.

[7] M. de Hoop and K. Sølna, Estimating a Green's function from fieldfield correlations in a random medium, SIAM J. Appl. Math., 69, (2009), pp. 909-932.

[8] J. Garnier and G. Papanicolaou, Passive sensor imaging using cross correlations of noisy signals in a scattering medium, SIAM J. Imaging Sciences, 2 (2009), pp. 396-437.

[9] J. Garnier and K. Solna Optimal Kirchhoff Migration of Cross Correlations, Submitted, (2009).

[10] P. Gouédard, L. Stehly, F. Brenguier, M. Campillo, Y. Colin de Verdière, E. Larose, L. Margerin, P. Roux, F. J. Sanchez-Sesma, N. M. Shapiro, and R. L. Weaver, Cross-correlation of random fields: mathematical approach and applications, Geophysical Prospecting, 56 (2008), pp. 375-393.

[11] O. I. Lobkis and R. L. Weaver, On the emergence of the Green's function in the correlations of a diffuse field, J. Acoustic. Soc. Am., 110 (2001), pp. 3011-3017.

[12] K. G. Sabra, P. Gerstoft, P. Roux, and W. Kuperman, Surface wave tomography from microseisms in Southern California, Geophys. Res. Lett., 32 (2005), L14311.

[13] L. Stehly, M. Campillo, and N. M. Shapiro, A study of the seismic noise from its long-range correlation properties, Geophys. Res. Lett., 111 (2006), B10306.

[14] H. Yao, R. D. van der Hilst, and M. V. de Hoop, Surface-wave array tomography in SE Tibet from ambient seismic noise and two-station analysis I. Phase velocity maps, Geophysical Journal International, 166 (2006), pp. 732-744. 